\title{
Minimal model linking two great mysteries: Neutrino mass and dark matter
}

\author{
Yasaman Farzan* \\ Institute for research in fundamental sciences (IPM), P.O. Box 19395-5531, Tehran, Iran
}

(Dated: November 7, 2018)

\begin{abstract}
We present an economic model that establishes a link between neutrino masses and properties of the dark matter candidate. The particle content of the model can be divided into two groups: light particles with masses lighter than the electroweak scale and heavy particles. The light particles, which also include the dark matter candidate, are predicted to show up in the low energy experiments such as ( $K \rightarrow \ell+$ missing energy), making the model testable. The heavy sector can show up at the LHC and may give rise to $\operatorname{Br}\left(\ell_{i} \rightarrow \ell_{j} \gamma\right)$ close to the present bounds. In principle, the new couplings of the model can independently be derived from the data from the LHC and from the information on neutrino masses and Lepton Flavor Violating (LFV) rare decays, providing the possibility of an intensive cross-check of the model.
\end{abstract}

PACS numbers:

\section{INTRODUCTION}

The nature of dark matter and the tiny neutrino masses are two great mysteries of modern particle physics and cosmology. A myriad of models have been proposed in the literature to explain either of these phenomena. Recently there have been some attempts to link these two phenomena [1, 2].

In Ref. [1], a scenario has been proposed that most economically accounts for the neutrino mass and simultaneously provides a dark matter candidate. The new particle content of the scenario is composed of a scalar field $\phi$ which plays the role of dark matter and two (or more) right-handed Majorana neutrinos, $N_{i}$. A $Z_{2}$ symmetry has been introduced under which $\phi$ and $N_{i}$ are odd but the standard model particles are even. The $Z_{2}$ symmetry stabilizes the lightest new particle, making it a suitable dark matter candidate. Moreover, the $Z_{2}$ symmetry forbids the $\epsilon_{\alpha \beta} \bar{N} H_{\alpha}^{T} L_{\beta}$ term in the Lagrangian so the neutrinos do not obtain a Dirac mass term.

Following [1], we shall call the scalar a SLIM. The scenario can be realized in two cases: i) real scalar; ii) complex scalar. In the present paper, we shall focus on the scenario with real SLIM. The scenario is based on the following low energy effective Lagrangian:

$$
\mathcal{L}=-g_{i \alpha} \bar{N}_{i} \nu_{\alpha L} \phi-\bar{N}_{j}^{c} \frac{\left(M_{N}\right)_{i j}}{2} N_{i}
$$

This Lagrangian at one loop level gives Majorana masses to the active neutrinos: $m_{\nu}=\left(g^{2} M_{N} / 16 \pi^{2}\right) \log \Lambda^{2} / M_{N}^{2}$. The dominant annihilation modes of the dark matter is into a neutrino or antineutrino pair: $\sigma_{t o t} \simeq \sigma(\phi \phi \rightarrow \nu \nu)+\sigma(\phi \phi \rightarrow$ $\bar{\nu} \bar{\nu})$. Under the assumption that the production of dark matter in the early universe was thermal, the dark matter abundance determines the annihilation cross section $\left(\left\langle\sigma_{t o t} v_{r}\right\rangle \sim 3 \cdot 10^{-26} \mathrm{~cm}^{3} / \mathrm{sec}\right)$, which is in turn given by the coupling $g$ and the masses of the right-handed neutrinos. The same parameters also determine the Majorana masses of active neutrinos: $\sigma_{t o t}=\left(g^{4} / 2 \pi\right)\left[m_{N}^{2} /\left(m_{\phi}^{2}+m_{N}^{2}\right)^{2}\right]$. Combining these two pieces of information, the parameters of the model can be restricted. In case of real scalar, the mass of the lightest right-handed neutrino is found to be in the range of few to $10 \mathrm{MeV}$. Remember that the scalar is taken to be even lighter than the right-handed neutrinos. On the other hand, a lower bound of few $\times 10^{-4}$ is derived on the coupling [1]. With the lower bound on the coupling and the upper bound on the masses of new particles, the scenario can be tested with experiments and observations that

*Electronic address: yasaman@theory.ipm.ac.ir 
are sensitive to rare but low energy processes; e.g., studies of supernova core collapse and searches for rare decays of light mesons such as the Kaon or pion [1].

The effective Lagrangian (11) is valid only in low energies and has to be embedded within a high energy theory which is invariant under $\mathrm{SU}(3) \times \mathrm{SU}(2)_{L} \times \mathrm{U}(1)$. Recently, a model has been proposed that embeds the scenario with a complex SLIM [3]. In [4], a model has been proposed that can be considered a realization of complex SLIM but the question of dark matter abundance has not been addressed in [4]. In the present paper, we propose a minimalistic model which embeds the scenario with a real SLIM.

The paper is organized as follows. In sect. II, we introduce the model. In sect. III we discuss the phenomenological implications of the model. The results are summarized in sect. IV.

\section{REAL SLIM}

The content of this model is composed of (1) an electroweak singlet, $\eta ;(2)$ two Majorana right-handed neutrinos, $N_{i}$ and (3) an electroweak doublet with nonzero hypercharge, $\Phi^{T}=\left[\begin{array}{ll}\phi^{0} & \phi^{-}\end{array}\right]$where $\phi^{0} \equiv\left(\phi_{1}+i \phi_{2}\right) / \sqrt{2}$ with real $\phi_{1}$ and $\phi_{2}$. Similarly to the scenario in [1], all these particles are odd under the $Z_{2}$ symmetry. The most general $Z_{2}$ even renormalizable Lagrangian involving only the scalars can be written as

$$
\begin{aligned}
\mathcal{L}= & -m_{\Phi}^{2} \Phi^{\dagger} \cdot \Phi-\frac{m_{s}^{2}}{2} \eta^{2}-\left(m_{\eta \Phi} \eta\left(H^{T}\left(i \sigma_{2}\right) \Phi\right)+\text { H.c. }\right) \\
& -\lambda_{1}\left|H^{T}\left(i \sigma_{2}\right) \Phi\right|^{2}-\operatorname{Re}\left[\lambda_{2}\left(H^{T}\left(i \sigma_{2}\right) \Phi\right)^{2}\right]-\lambda_{3} \eta^{2} H^{\dagger} H-\lambda_{4} \Phi^{\dagger} \cdot \Phi H^{\dagger} \cdot H \\
& -\frac{\lambda_{1}^{\prime}}{2}\left(\Phi^{\dagger} \cdot \Phi\right)^{2}-\frac{\lambda_{2}^{\prime}}{2} \eta^{4}-\lambda_{3}^{\prime} \eta^{2} \Phi^{\dagger} \cdot \Phi \\
& -m_{H}^{2} H^{\dagger} \cdot H-\frac{\lambda}{2}\left(H^{\dagger} \cdot H\right)^{2}
\end{aligned}
$$

Certain conditions on the parameters of the model should be satisfied so that the theory becomes stable in the sense that when a combination of the fields goes to infinity, the potential remains positive [5]. Some of these conditions are

$$
\lambda_{1}^{\prime}, \lambda_{2}^{\prime}>0, \lambda_{3}^{\prime}>-\left(\lambda_{1}^{\prime} \lambda_{2}^{\prime}\right)^{1 / 2}, \lambda_{3}>-\left(\lambda \lambda_{2}^{\prime}\right)^{1 / 2}
$$

and

$$
\lambda_{1}-\left|\lambda_{2}\right|+\lambda_{4}>-\left(\lambda \lambda_{1}^{\prime}\right)^{1 / 2}
$$

For simplicity we take the Lagrangian to be CP-conserving which means $m_{\eta \Phi}$ and $\lambda_{2}$ are both real. As we shall see below, after electroweak symmetry breaking, the term $m_{\eta \Phi}$ mixes $\eta$ with the CP-even neutral component of $\Phi$ (i.e., $\left.\phi_{1}\right)$. The lightest new particle is a linear combination of $\eta$ and $\phi_{1}$ with a dominant contribution from $\eta$. Because of the $Z_{2}$ symmetry, such a combination is stable and plays the role of the dark matter [i.e., the role of $\phi$ in Ref. [1]; see Eq. (11)].

The Lagrangian involving the right-handed neutrinos in the mass basis of right-handed neutrinos is

$$
\mathcal{L}=-g_{i \alpha} \bar{N}_{i} \Phi^{\dagger} \cdot L_{\alpha}-\frac{M_{i}}{2} \bar{N}_{i}^{c} N_{i}
$$

where $L_{\alpha}$ is the lepton doublet of flavor $\alpha: L_{\alpha}^{T}=\left(\nu_{L \alpha} \ell_{L \alpha}^{-}\right)$.

After electroweak symmetry breaking (i.e., setting $\left.H^{T}=\left(0 v_{H} / \sqrt{2}\right)\right)$, the mass terms will be of form

$$
\begin{aligned}
\mathcal{L}_{m} & =-m_{\phi^{-}}^{2}\left|\phi^{-}\right|^{2}-\frac{m_{\phi_{2}}^{2}}{2} \phi_{2}^{2} \\
& -\frac{m_{\eta}^{2}}{2} \eta^{2}-\frac{m_{\phi_{1}}^{2}}{2} \phi_{1}^{2}-m_{\eta \Phi} v_{H} \phi_{1} \eta
\end{aligned}
$$


where

$$
\begin{aligned}
m_{\phi^{-}}^{2} & =m_{\Phi}^{2}+\lambda_{4} \frac{v_{H}^{2}}{2} \\
m_{\eta}^{2} & =m_{s}^{2}+\lambda_{3} \frac{v_{H}^{2}}{2} \\
m_{\phi_{1}}^{2} & =m_{\Phi}^{2}+\lambda_{1} \frac{v_{H}^{2}}{2}+\lambda_{2} \frac{v_{H}^{2}}{2} \\
m_{\phi_{2}}^{2} & =m_{\Phi}^{2}+\lambda_{1} \frac{v_{H}^{2}}{2}-\lambda_{2} \frac{v_{H}^{2}}{2} .
\end{aligned}
$$

The parameters can be tuned such that the squares of all mass eigenvalues become positive. This means that other than the Higgs field, none of the scalars develops a vacuum expectation value so the $Z_{2}$ symmetry remains unbroken and moreover neutrinos do not acquire any Dirac mass at the tree level. $\phi^{-}$and $\phi_{2}$ are both mass eigenvalues whose masses can be readily read respectively from Eq. (6) and Eq. (9). To avoid the bounds from direct searches, $m_{\phi^{-}}^{2}$ and $m_{\phi_{2}}^{2}$ are taken above the electroweak scale. Assuming the couplings of Higgs are relatively small (i.e., $\lesssim 0.1$ ), this means $m_{\Phi}^{2}$, and consequently $m_{\phi_{2}}^{2}, m_{\phi_{1}}^{2}$ and $m_{\phi^{-}}^{2}$, are of order of (or larger than) $O\left((100 \mathrm{GeV})^{2}\right)$. However, $m_{\eta}^{2}$ can be below the electroweak scale. The mass eigenstates are

$$
\left[\begin{array}{l}
\delta_{1} \\
\delta_{2}
\end{array}\right]=\left[\begin{array}{cc}
\cos \alpha & -\sin \alpha \\
\sin \alpha & \cos \alpha
\end{array}\right]\left[\begin{array}{c}
\eta \\
\phi_{1}
\end{array}\right]
$$

with

$$
\begin{aligned}
\tan 2 \alpha & =\frac{2 v_{H} m_{\eta \Phi}}{m_{\phi_{1}}^{2}-m_{\eta}^{2}} \\
m_{\delta_{1}}^{2} & \simeq m_{\eta}^{2}-\frac{\left(m_{\eta \Phi} v_{H}\right)^{2}}{m_{\phi_{1}}^{2}-m_{\eta}^{2}} \\
m_{\delta_{2}}^{2} & \simeq m_{\phi_{1}}^{2}+\frac{\left(m_{\eta \Phi} v_{H}\right)^{2}}{m_{\phi_{1}}^{2}-m_{\eta}^{2}},
\end{aligned}
$$

where in the last two equations we have used $\left(m_{\eta \Phi} v_{H}\right)^{2} /\left(m_{\phi_{1}}^{2}-m_{\eta}^{2}\right)^{2} \ll 1$. The couplings of the mass eigenvalues $\delta_{1}$ and $\delta_{2}$ to $\bar{N}_{i} \nu_{L \alpha}$ are listed in Table [. We are interested in the following range:

$$
m_{\delta_{1}}^{2}<m_{N_{1}}^{2} \ll m_{\delta_{2}}^{2} \simeq m_{\phi_{2}}^{2} \simeq m_{\phi^{-}}^{2} \sim m_{\text {electroweak }}^{2}
$$

and

$$
\left|\frac{m_{\phi_{2}}^{2}-m_{\delta_{2}}^{2}}{m_{\phi_{2}}^{2}+m_{\delta_{2}}^{2}}\right| \simeq\left|-\frac{\lambda_{2}}{2} \frac{v_{H}^{2}}{m_{\phi_{2}}^{2}}-\frac{\sin ^{2} \alpha}{2}\right| \ll 1 .
$$

$\delta_{1}$, being the lightest $Z_{2}$-odd particle, is the dark matter candidate. Using the couplings in Table \. we find

$$
\left\langle\sigma\left(\delta_{1} \delta_{1} \rightarrow \nu_{L \alpha} \nu_{L \beta}\right) v_{r}\right\rangle=\left\langle\sigma\left(\delta_{1} \delta_{1} \rightarrow \bar{\nu}_{L \alpha} \bar{\nu}_{L \beta}\right) v_{r}\right\rangle=\frac{\sin ^{4} \alpha}{8 \pi}\left|\sum_{i} \frac{g_{i \alpha} g_{i \beta} m_{N_{i}}}{m_{\delta_{1}}^{2}+m_{N_{i}}^{2}}\right|^{2} .
$$

As seen from this formula (and as discussed in detail in [1]), the lightest $N_{i}$ is in general expected to dominate the annihilation cross section so we obtain

$$
\operatorname{Max}\left[g_{1 \beta}\right] \sin \alpha \sim 5 \times 10^{-4}\left(\frac{m_{N_{1}}}{\mathrm{MeV}}\right)^{1 / 2}\left(\frac{\left\langle\sigma v_{r}\right\rangle}{3 \cdot 10^{-26} \mathrm{~cm}^{3} \mathrm{sec}^{-1}}\right)^{1 / 4}\left(1+\frac{m_{\delta_{1}}^{2}}{m_{N_{1}}^{2}}\right)^{1 / 2} .
$$

As discussed in [1], the terms in Eq. (3) give a Majorana mass to neutrinos through a one loop diagram. Using Eq. (2) of [1], we find

$$
\begin{aligned}
\left(m_{\nu}\right)_{\alpha \beta}= & \sum_{i} \frac{g_{i \alpha} g_{i \beta}}{32 \pi} m_{N_{i}}\left[\sin ^{2} \alpha\left(\frac{m_{\delta_{2}}^{2}}{m_{N_{i}}^{2}-m_{\delta_{2}}^{2}} \log \frac{m_{N_{i}}^{2}}{m_{\delta_{2}}^{2}}-\frac{m_{\delta_{1}}^{2}}{m_{N_{i}}^{2}-m_{\delta_{1}}^{2}} \log \frac{m_{N_{i}}^{2}}{m_{\delta_{1}}^{2}}\right)\right. \\
& \left.+\frac{m_{\phi_{2}}^{2}}{m_{N_{i}}^{2}-m_{\phi_{2}}^{2}} \log \frac{m_{N_{i}}^{2}}{m_{\phi_{2}}^{2}}-\frac{m_{\delta_{2}}^{2}}{m_{N_{i}}^{2}-m_{\delta_{2}}^{2}} \log \frac{m_{N_{i}}^{2}}{m_{\delta_{2}}^{2}}\right] .
\end{aligned}
$$




\begin{tabular}{|l|l|l|}
\hline particle & mass & coupling to $\bar{N}_{i} \nu_{\alpha}$ \\
\hline \hline$\delta_{1}$ & $m_{\delta_{1}}$ & $-\frac{\sin \alpha}{\sqrt{2}} g_{i \alpha}$ \\
$\delta_{2}$ & $m_{\delta_{2}}$ & $\frac{\cos \alpha}{\sqrt{2}} g_{i \alpha}$ \\
$\phi_{2}$ & $m_{\phi_{2}}$ & $\frac{i}{\sqrt{2}} g_{i \alpha}$ \\
\hline
\end{tabular}

TABLE I: Neutral scalars of the model .

Notice that neutrino mass and dark matter annihilation are given by the same coupling. That is why these two seemingly different quantities are linked. Such a relation is a feature of the scenario in [1] which is embedded within the present model.

Taking $m_{\delta_{1}}^{2}<m_{N_{i}}^{2} \ll m_{\delta_{2}}^{2}$ and using Eq. (15), we find

$$
\left(m_{\nu}\right)_{\alpha \beta} \simeq \sum_{i} \frac{g_{i \alpha} g_{i \beta}}{32 \pi} m_{N_{i}}\left[\sin ^{2} \alpha\left(\frac{m_{\delta_{2}}^{2}}{m_{N_{i}}^{2}-m_{\delta_{2}}^{2}} \log \frac{m_{N_{i}}^{2}}{m_{\delta_{2}}^{2}}-\frac{m_{\delta_{1}}^{2}}{m_{N_{i}}^{2}-m_{\delta_{1}}^{2}} \log \frac{m_{N_{i}}^{2}}{m_{\delta_{1}}^{2}}-1\right)-\lambda_{2} \frac{v_{H}^{2}}{m_{\phi_{2}}^{2}}\right] .
$$

Three situations are imaginable: (1) $\lambda_{2} v_{H}^{2} /\left(m_{\phi_{2}}^{2}\right) \gg \sin ^{2} \alpha \log \left(m_{\delta_{2}}^{2} / m_{N}^{2}\right) ;(2) \lambda_{2} v_{H}^{2} /\left(m_{\phi_{2}}^{2}\right) \sim \sin ^{2} \alpha \log \left(m_{\delta_{2}}^{2} / m_{N}^{2}\right)$; and (3) $\lambda_{2} v_{H}^{2} /\left(m_{\phi_{2}}^{2}\right) \ll \sin ^{2} \alpha \log \left(m_{\delta_{2}}^{2} / m_{N}^{2}\right)$. If we proceed with case (1) and combine Eq. (17) with Eq. (19), we find that the preassumption (i.e., $\left.\lambda_{2} v_{H}^{2} / m_{\phi_{2}}^{2} \gg \sin ^{2} \alpha \log \left(m_{N}^{2} / m_{\delta_{2}}^{2}\right)\right)$ implies $m_{N_{1}} \ll 1 \mathrm{MeV}$ which might be at odds with nucleosynthesis data [6]. Situations (2) and (3) imply

$$
m_{N_{1}} \sim(1 \mathrm{MeV})\left(\frac{3 \cdot 10^{-26} \mathrm{~cm}^{3} \mathrm{sec}^{-1}}{\left\langle\sigma v_{r}\right\rangle}\right)^{1 / 4}\left(\frac{25}{\log m_{N_{1}}^{2} / m_{\delta_{2}}^{2}}\right)^{1 / 2}\left(\frac{m_{\nu}}{\sqrt{\Delta m_{a t m}^{2}}}\right)^{1 / 2}\left(1+\frac{m_{\delta_{1}}^{2}}{m_{N_{1}}^{2}}\right)^{-1 / 2}
$$

which is the same conclusion reached in Ref. [1]:

$$
m_{\delta_{1}}<m_{N_{1}} \sim \text { few } \mathrm{MeV},
$$

and can be made compatible with the nucleosynthesis data 7]. As discussed in [1], in order to reproduce the neutrino data at least two $N_{i}$ should be present. Within minimalistic scenario with only two $N_{i}$, one of the active neutrino mass eigenvalues vanishes which means the neutrino mass scheme is hierarchical. Assuming that annihilation $\delta_{1} \delta_{1} \rightarrow \nu_{L} \nu_{L}$ is dominated by $N_{1}$, the only bound on the parameters comes from the neutrino mass matrix. Taking $m_{\delta_{2}}^{2} \simeq m_{\phi_{2}}^{2} \simeq m_{\phi^{-}}^{2} \sim m_{\text {electroweak }}^{2}$, we obtain

$$
\left\{\begin{array}{lc}
g_{2 \alpha} \sim 2 \times 10^{-4}\left(\frac{m_{\nu}}{\sqrt{\Delta m_{a t m}^{2}}}\right)^{1 / 2}\left(\frac{m_{N_{2}}}{10 \mathrm{MeV}}\right)^{-1 / 2} \frac{1}{\sin \alpha} & 1 \mathrm{MeV}<m_{N_{2}} \ll m_{\delta_{2}} \\
g_{2 \alpha} \sim 7 \times 10^{-6}\left(\frac{m_{\nu}}{\sqrt{\Delta m_{a t m}^{2}}}\right)^{1 / 2}\left(\frac{m_{N_{2}}}{100 \mathrm{GeV}}\right)^{1 / 2} \frac{1}{\sin \alpha} & m_{\delta_{2}} \ll m_{N_{2}} \ll m_{\delta_{2}} \sin \alpha / \lambda_{2} \\
g_{2 \alpha} \sim 4 \times 10^{-6}\left(\frac{m_{\nu}}{\sqrt{\Delta m_{\text {atm }}^{2}}}\right)^{1 / 2}\left(\frac{m_{N_{2}}}{100 \mathrm{GeV}}\right)^{-1 / 2} \frac{1}{\sqrt{\lambda_{2}}} & m_{\delta_{2}} \sin \alpha / \lambda_{2} \ll m_{N_{2}}
\end{array}\right.
$$

Notice that neither the constraints from the neutrino data nor the one from the dark matter abundance yields any bound on the masses of the components of $\Phi$, i.e., $m_{\phi^{-}}, m_{\phi_{1}}$ or $m_{\phi_{2}}$. In principle, as long as $\sin \alpha \sim v_{H} m_{\eta \phi} /\left(m_{\phi_{1}}^{2}-m_{\eta}^{2}\right)$ remains smaller than few $\times 10^{-4}$ (see Eq. (17)), the components of $\Phi$ can be arbitrarily heavy. Notice however that for $m_{\phi_{1}}^{2} / m_{\text {Electroweak }}^{2} \rightarrow \infty, m_{\eta \phi}$ also grows as $\sin \alpha m_{\phi_{1}}^{2} / v_{H}$. Moreover for $m_{\phi_{1}}^{2} \gg m_{\text {electroweak }}^{2}$, from Eq. (11) we observe that in order to maintain $m_{\delta_{1}}^{2}$ around $(1 \mathrm{MeV})^{2}$, a fine tuned cancelation between $m_{\eta}^{2}$ and $\left(m_{\eta \Phi} v_{H}\right)^{2} /\left(m_{\phi_{1}}^{2}-m_{\eta}^{2}\right) \sim$ $\sin ^{2} \alpha\left(m_{\phi_{1}}^{2}-m_{\eta}^{2}\right)$ is required. The degree of fine tuning is $m_{\delta_{1}}^{2} /\left(\sin ^{2} \alpha m_{\phi_{1}}^{2}\right) \simeq 10^{-4}(1 \mathrm{TeV})^{2} / m_{\phi_{1}}^{2}$ so to keep the degree of fine tuning agreeable, the range $m_{\phi_{1}}^{2} \ll(\mathrm{TeV})^{2}$ is more desirable.

After replacing $g \rightarrow g \sin \alpha / \sqrt{2}$, all discussion in Ref. [1] on the falsifiability of the model via low energy experiments can be repeated here, too. In addition to the phenomenological implications discussed in [1], the heavy states will cause a number of potentially observable effects which we discuss in the next section. 


\section{PHENOMENOLOGICAL IMPLICATIONS}

In Ref. [1, 8], a number of phenomenological consequences of the low energy content of the scenario have been discussed. The same consideration applies when the scenario is embedded within a model.

Large scale structure arguments imply that the dark matter candidate has to be heavier than few keV [1, 9]. Although our dark matter candidate can affect the supernova evolution, it can be accommodated within current uncertainties [1]. Detection of future supernova explosions will be a powerful probe for this scenario. Finally, since there are new light particles $N_{i}$ and $\delta_{1}$ which couple to ordinary neutrinos, they can show up in light meson decays as missing energy signal $\left(K(\pi) \rightarrow \ell N_{i} \delta_{1}\right)$. Present bounds on the coupling [10] are too weak to rule out our model [1]. Eventually by improving the bound on $K \rightarrow \ell+$ missing energy and $\pi \rightarrow \ell+$ missing energy, the condition in (17) can be tested [11]. Thus, a careful analysis of the KLOE results is imperative for testing this model.

From Eq. (10), we observe that while real singlet, $\eta$, and the CP-even component of $\Phi, \phi_{1}$ mix, the CP-odd component, $\phi_{2}$ is decoupled and does not enter the mixing matrix. This is expected because we took the Lagrangian to be CP-even. Had we allowed $m_{\eta \phi}$ and $\lambda_{2}$ to be complex, a mixing between $\eta$ and $\phi_{2}$ would also appear. The coupling of $\phi^{0}=\left(\phi_{1}+i \phi_{2}\right) / \sqrt{2}$ to $Z$ boson is of the following form:

$$
\left(\phi_{2} \partial_{\mu} \phi_{1}-\phi_{1} \partial_{\mu} \phi_{2}\right) Z^{\mu}
$$

The absence of a component of $\phi_{2}$ in $\delta_{1}$ has several consequences: 1 ) Since $\phi_{2}$ is heavy, no new decay mode for the $Z$ boson appears. 2) The interaction of dark matter with electrons and nuclei does not take place at tree level, making direct detection as well as trapping in dense environments such as the Sun more challenging. Moreover, the mass of the dark matter candidate in this model is too small to lead to a detectable recoil energy in direct dark matter search experiments. Through couplings to the Higgs boson (i.e., $\lambda_{i}$ ), the dark matter can interact with nuclei. If the couplings are relatively large, dark matter can be trapped inside the Sun. Relatively high abundance of dark matter in the halo can give rise to a neutrino flux of few $\mathrm{MeV}$ potentially detectable at Super-Kamiokande. The corresponding bound has been studied in [12] but the present bound is too weak to probe $\left\langle\sigma v_{r}\right\rangle \sim 3 \cdot 10^{-26} \mathrm{~cm}^{3} \mathrm{sec}^{-1}$.

In addition to the effects caused by light particles of the model (i.e., $N_{i}$ and $\delta_{1}$ ), due to the presence of the heavier particles within the present model, a number of new effects will appear which we discuss below.

\section{A. Annihilation into electron positron pair}

In the present model, the annihilation of dark matter to the $e^{-} e^{+}$pair can take place only at the loop level. There are two one-loop diagrams contributing to this annihilation. In one of these two, the vertices involving $\delta_{1}$ are of the form $-\left(g_{i e} \sin \alpha / \sqrt{2}\right) \delta_{1} \bar{N}_{i} \nu_{e}$ and in the other, only the gauge couplings enter. Depending on the range of parameters, either of the following contributions to the annihilation cross section can be dominant:

$$
\left\langle\sigma v_{r}\right\rangle \sim\left(\frac{e^{4}}{16 \pi^{2} \sin ^{4} \theta_{W}}\right)^{2} \frac{\left(m_{\delta_{1}} v_{r}\right)^{2}}{8 \pi m_{W}^{4}} \sin ^{4} \alpha \quad \text { or } \quad \sim\left(\frac{e^{2} \sum_{i}\left|g_{i e}\right|^{2}}{16 \pi^{2} \sin ^{2} \theta_{W}}\right)^{2} \frac{\left(m_{\delta_{1}} v_{r}\right)^{2}}{8 \pi m_{W}^{4}} \sin ^{4} \alpha .
$$

Since SLIMs are scalars and the annihilation into an electron positron pair does not flip the chirality, the annihilation is a $p$-wave process so the cross section is proportional to the velocity square $\left(v_{r}^{2}\right)$. In order to explain the 511 keV line from the center of the galaxy the ratio $\sigma$ (Dark Matter + Dark Matter $\left.\rightarrow e^{-}+e^{+}\right) / \sigma_{t o t}$ has to be of order of $10^{-4}$. The loop suppression factor $\left[\right.$ i.e., $\left.e^{4} /\left(16 \pi^{2} \sin ^{2} \theta_{w}\right)^{4}\right]$ is smaller than this amount so the 511 keV line cannot be explained within this model. Moreover, because of the low annihilation cross section, we do not expect any detectable photon radiation from the emitted $e^{-} e^{+}$pairs. Notice that the annihilation of non-relativistic SLIMs into $\mu^{+} \mu^{-}$cannot take place because of the low mass of the particles.

\section{B. Annihilation into photon pair}

There are several one-loop diagrams that contribute to the annihilation into a photon pair. Each diagram is divergent but the divergences cancel each other and at the end of the day, the cross section can be written as

$$
\sigma\left(\delta_{1} \delta_{1} \rightarrow \gamma \gamma\right) \sim \frac{e^{8} \sin ^{4} \alpha}{8 \pi\left(16 \pi^{2}\right)^{2} \cos ^{4} \theta_{W}} \frac{m_{\delta_{1}}^{2}}{m_{W}^{4}} \sim \text { few } \times 10^{-41}\left(\frac{M_{\delta_{1}}}{\mathrm{MeV}}\right)^{2} \sin ^{4} \alpha \mathrm{cm}^{3} / \mathrm{sec}
$$


The value of annihilation cross section is too small for the Fermi telescope (formerly known as GLAST) to detect a signal for annihilation into a photon pair (see, e.g., Fig 4 of [13]).

\section{Lepton Flavor Violating rare decay}

In both of the models presented in this paper, there is a charged scalar that couples to the charged leptons through $g_{i \alpha} \bar{N}_{i} \ell_{L \alpha} \phi^{-}$. This coupling leads to the Lepton Flavor Violating rare decays, $\mu \rightarrow e \gamma, \tau \rightarrow \mu \gamma$ and $\tau \rightarrow e \gamma$. Using the formulas in [14], we find

$$
\Gamma\left(\ell_{\alpha} \rightarrow \ell_{\beta} \gamma\right)=\frac{m_{\alpha}^{3}}{16 \pi}\left|\sigma_{R}\right|^{2}
$$

where

$$
\sigma_{R}=\sum_{i} g_{i \alpha} g_{i \beta}^{*} \frac{i e m_{\alpha}}{16 \pi^{2} m_{\phi^{-}}^{2}} K\left(t_{i}\right)
$$

where $t_{i}=m_{N_{i}}^{2} / m_{\phi^{-}}^{2}$ and

$$
K\left(t_{i}\right)=\frac{2 t_{i}^{2}+5 t_{i}-1}{12\left(t_{i}-1\right)^{3}}-\frac{t_{i}^{2} \log t_{i}}{2\left(t_{i}-1\right)^{4}} .
$$

The following three limits are of interest: $(1) t_{i} \rightarrow 0$ for which $K\left(t_{i}\right) \rightarrow 1 / 12$; (2) $t_{i} \rightarrow \infty$ which implies $K\left(t_{i}\right) \rightarrow 1 /\left(6 t_{i}\right)$ and (3) $t_{i} \sim 1$ which implies $K\left(t_{i}\right) \sim 1$.

As discussed earlier, the mass of at least one of $N_{i}$ has to be around $1 \mathrm{MeV}$. The mass of the other $N_{i}$ can be larger. As long as $m_{N_{2}}^{2} \ll m_{\phi^{-}}^{2}$, we can write

$$
\begin{gathered}
\operatorname{Br}(\mu \rightarrow e \gamma) \sim 2 \times 10^{-4}\left|\sum_{i} g_{\mu i} g_{e i}^{*}\right|^{2}\left(\frac{100 \mathrm{GeV}}{m_{\phi^{-}}}\right)^{4} \\
\operatorname{Br}\left(\tau \rightarrow \ell_{\alpha} \gamma\right) \sim 5 \times 10^{-5}\left|\sum_{i} g_{i \tau} g_{i \alpha}^{*}\right|^{2}\left(\frac{100 \mathrm{GeV}}{m_{\phi^{-}}}\right)^{4} .
\end{gathered}
$$

From PDG booklet [15], we read

$$
\begin{gathered}
\operatorname{Br}(\mu \rightarrow e \gamma)<1.2 \times 10^{-11} \\
\operatorname{Br}(\tau \rightarrow e \gamma)<1.1 \times 10^{-7} \\
\operatorname{Br}(\tau \rightarrow \mu \gamma)<6.8 \times 10^{-8}
\end{gathered}
$$

From Eqs. (17/21), we realize that the present bounds can be readily satisfied. A particularly interesting range is the following:

$$
m_{\phi^{-}} \sim 100 \mathrm{GeV} \quad g_{i \mu}, g_{i \tau} \sim \text { few } \times 10^{-2} \text { and } g_{i e} \sim \text { few } \times 10^{-3},
$$

which leads to the LFV rates detectable in near future [16]. Notice that a little hierarchy of $g_{i e} \sim$ $\left(\Delta m_{\text {sol }}^{2} / \Delta m_{\text {atm }}^{2}\right)^{1 / 2} g_{i \mu} \sim 0.1 g_{i \mu}$ is desirable for normal hierarchical mass scheme.

In future if these LFV rare decays are discovered, it will be possible to derive information on the flavor structure of the couplings. By combining the information from the flavor structure of the neutrino mass matrix with the information from LFV searches the models can be probed [11].

\section{Magnetic dipole moment of the muon}

In the models presented in this paper, the magnetic dipole moment of the muon obtains a contribution via coupling $g_{i \mu} \bar{N}_{i} \mu\left(\phi^{-}\right)^{\dagger}$ :

$$
\delta \frac{g-2}{2}=\sum_{i} \frac{\left|g_{i \mu}\right|^{2}}{16 \pi^{2}} \frac{m_{\mu}^{2}}{m_{\phi^{-}}^{2}} K\left(t_{i}\right),
$$


where $K\left(t_{i}\right)$ is defined in Eq. (24). For $t_{i} \ll 1$, which is what we expect for our model, we can write

$$
\delta \frac{g-2}{2}=5 \times 10^{-12} \frac{\sum_{i}\left|g_{i \mu}\right|^{2}}{10^{-2}}\left(\frac{100 \mathrm{GeV}}{m_{\phi^{-}}^{2}}\right)^{2}
$$

so the present bounds can be readily satisfied. The predicted value is below the present bound by two orders of magnitude.

\section{E. Dark matter self interaction}

The $\lambda_{i}^{\prime}$ couplings can give rise to self interaction of the SLIM particles:

$$
\left\langle\sigma\left(\delta_{1} \delta_{1} \rightarrow \delta_{1} \delta_{1}\right) v\right\rangle \sim \operatorname{Max}\left[\frac{\left|\lambda_{1}^{\prime}\right|^{2} \sin ^{4} \alpha}{8 \pi m_{\delta_{1}}^{2}}, \frac{\left|\lambda_{2}^{\prime}\right|^{2} \cos ^{4} \alpha}{8 \pi m_{\delta_{1}}^{2}}, \frac{\left|\lambda_{3}^{\prime}\right|^{2} \sin ^{2} \alpha \cos ^{2} \alpha}{8 \pi m_{\delta_{1}}^{2}}\right] .
$$

From considering the merging of the galaxy clusters, bounds on $\sigma / m_{D M}$ have been derived [17]: $\sigma / m_{D M} \lesssim 1 \mathrm{~cm}^{2} / \mathrm{g}$. The bound implies

$$
\left|\lambda_{1}^{\prime}\right|^{2} \sin ^{4} \alpha,\left|\lambda_{2}^{\prime}\right|^{2} \cos ^{4} \alpha,\left|\lambda_{3}^{\prime}\right|^{2} \sin ^{2} \alpha \cos ^{2} \alpha \lesssim 10^{-4}
$$

There have been some suggestions to employ self interaction of dark matter with $\sigma / m_{D M}=(0.5-5) \mathrm{cm}^{2} / \mathrm{g}$ to explain the observed mass profiles of the galaxies [18]. The possibility can be readily accommodated within the present model.

\section{F. Production at LHC}

Last but not least, the components of doublet $\Phi$ can in principle be produced at the LHC through electroweak interactions in pairs. All the new particles will eventually decay into SLIM which appears as missing energy signal at the detector.

Remember that at least one of the right-handed neutrinos has to be lighter than the doublet. As a result, $\phi^{-}$can decay into $\ell_{\beta}^{-}$and $N_{i}$

$$
\Gamma\left(\phi^{-} \rightarrow \ell_{\beta}^{-} N_{i}\right) \propto\left|g_{i \beta}\right|^{2} .
$$

Thus, by studying the two body decay mode into a charged lepton, the couplings to light right-handed neutrinos can be determined. The same couplings enter the neutrino mass matrix and the formulas for the rates of the LFV rare decays so the model can be cross checked by studying the correlations [11]. The light $N_{i}$ will eventually decay into a SLIM and ordinary neutrinos: $N_{i} \rightarrow \nu \delta_{1}$. Both of the decay products escape detection and appear as missing energy signal. As discussed in the end of sect. II there is no upper bound on the masses of the components of the doublet but from the fine tuning considerations, mass scale of $100 \mathrm{GeV}$ is more desirable. Thus, if the LHC does not find any signal for such a doublet, the model cannot be falsified however, it will require a higher degree of fine tuning.

Within this scenario Higgs can have invisible decay modes $H \rightarrow \delta_{1} \delta_{1}$. If $\operatorname{Max}\left[\cos ^{2} \alpha \lambda_{3}, \sin ^{2} \alpha \lambda_{i}\right.$ with $\left.i \neq 3\right] \gtrsim m_{b} / v_{H} \simeq$ 0.02 , this decay mode can dominate over the standard decay mode $H \rightarrow b \bar{b}$.

\section{CONCLUSIONS}

We have proposed a model that encompasses the scenario introduced in [1] for linking dark matter problem with the neutrino mass puzzle. The model inherits the features of the scenario: It contains a scalar (SLIM) and one (or more) right-handed neutrino(s) with masses lower than $O(1 \mathrm{MeV})$. As in Ref. 1], combining data on neutrino mass scale with dark matter abundance puts a lower bound on the couplings between the right-handed neutrinos with the SLIM which makes the model testable by low energy experiments. Since in the model the mass of the dark matter is much smaller than the nucleon mass, we expect null result in direct searches for the dark matter which are based on measuring the recoil energy of scattering of dark matter particles off nuclei in a sample. 
The heavy particles of the model which consist of the components of a scalar doublet can in principle be produced at the accelerators. The charged component of the doublet, $\phi^{-}$, which couples to charged leptons via the same LFV couplings that enter the neutrino mass matrix, can give rise to LFV processes, $\ell_{i} \rightarrow \ell_{j} \gamma$ at one loop level. Studying the decay modes of $\phi^{-}$, the flavor structure of its Yukawa coupling can be derived and can be tested against the information obtained from flavor structure of the neutrino mass matrix. We have not derived any upper bound on the masses of the doublet so if the LHC reports a null result, the model will not be ruled out. We have however shown that the fine tuning arguments points towards low mass scale doublets and therefore towards a rich phenomenology at the LHC.

\section{Acknowledgments}

I would like to thank S. Pascoli, T. Hambye, M. Schmidt and M. M. Sheikh-Jabbari for useful discussions.

[1] C. Boehm, Y. Farzan, T. Hambye, S. Palomares-Ruiz and S. Pascoli, Phys. Rev. D 77 (2008) 043516.

[2] L. M. Krauss, S. Nasri and M. Trodden, Phys. Rev. D 67 (2003) 085002 arXiv:hep-ph/0210389; K. Cheung and O. Seto, Phys. Rev. D 69 (2004) 113009 arXiv:hep-ph/0403003; T. Asaka, S. Blanchet and M. Shaposhnikov, Phys. Lett. B 631 (2005) 151 arXiv:hep-ph/0503065; E. J. Chun and H. B. Kim, JHEP 0610 (2006) 082 arXiv:hep-ph/0607076; J. Kubo and D. Suematsu, Phys. Lett. B 643 (2006) 336 arXiv:hep-ph/0610006; J. Kubo, E. Ma and D. Suematsu, Phys. Lett. B 642 (2006) 18 arXiv:hep-ph/0604114; T. Hambye, K. Kannike, E. Ma and M. Raidal, Phys. Rev. D 75 (2007) 095003 arXiv:hep-ph/0609228; N. Sahu and U. Sarkar, Phys. Rev. D 78, 115013 (2008) arXiv:0804.2072 [hep-ph]].

[3] Y. Farzan, S. Pascoli and M. Schmidt, arXiv:1005.5323 [hep-ph]; to appear in JHEP.

[4] E. Ma, Phys. Rev. D 73 (2006) 077301 arXiv:hep-ph/0601225].

[5] H. E. Haber, Phys. Rev. D 67 (2003) 075019 arXiv:hep-ph/0207010.

[6] S. Hannestad and G. G. Raffelt, JCAP 0611 (2006) 016 arXiv:astro-ph/0607101].

[7] P. D. Serpico and G. G. Raffelt, Phys. Rev. D 70 (2004) 043526 arXiv:astro-ph/0403417.

[8] C. Boehm and R. Schaeffer, arXiv:astro-ph/0410591; C. Boehm, P. Fayet and R. Schaeffer, Phys. Lett. B 518 (2001) 8 arXiv:astro-ph/0012504.

[9] U. Seljak, A. Makarov, P. McDonald and H. Trac, Phys. Rev. Lett. 97 (2006) 191303 arXiv:astro-ph/0602430; A. Boyarsky, J. Lesgourgues, O. Ruchayskiy and M. Viel, JCAP 0905 (2009) 012 arXiv:0812.0010 [astro-ph]].

[10] D. I. Britton et al., Phys. Rev. D 49 (1994) 28; V. D. Barger, W. Y. Keung and S. Pakvasa, Phys. Rev. D 25 (1982) 907 ; G. B. Gelmini, S. Nussinov and M. Roncadelli, Nucl. Phys. B 209 (1982) 157.

[11] Y. Farzan, arXiv:1009.1234 [hep-ph].

[12] S. Palomares-Ruiz and S. Pascoli, Phys. Rev. D 77 (2008) 025025 arXiv:0710.5420 [astro-ph]].

[13] S. Profumo, Phys. Rev. D 78, 023507 (2008) arXiv:0806.2150 [hep-ph]].

[14] L. Lavoura, Eur. Phys. J. C 29 (2003) 191 arXiv:hep-ph/0302221.

[15] C. Amsler et al. [Particle Data Group], Phys. Lett. B 667 (2008) 1.

[16] http://meg.web.psi.ch/index.html http://superb.kek.jp/; M. A. Giorgi, J. Phys. Conf. Ser. 171 (2009) 012022.

[17] S. W. Randall, M. Markevitch, D. Clowe, A. H. Gonzalez and M. Bradac, arXiv:0704.0261 [astro-ph].

[18] R. Dave, D. N. Spergel, P. J. Steinhardt and B. D. Wandelt, Astrophys. J. 547 (2001) 574 arXiv:astro-ph/0006218; 